\section{Measles Immunization: Worth Considering Containment Strategy for SARS-CoV-2 Global Outbreak}

Published online: March 29, 2020; PII: S097455911600153

Data on individuals aged 18 years and under reported by the World Health Organization (WHO) China Joint Mission on COVID-19 suggest that there is a relatively low attack rate in this age group (2.4\% of all reported cases). These observations are in agreement with epidemiological pattern of 2003 Coronavirus SARS-CoV outbreak when 8,098 cases of SARS were diagnosed worldwide and only $<5 \%$ of all cases were diagnosed in patients $<18$ years of age [1]. 2012 MERS-Coronavirus outbreak reported few cases of MERS-CoV in children and it remained mainly a disease of adults [2].

Measles Mumps Rubella (MMR) vaccine has been thought to protect against other viral causes of respiratory diseases as well. Although satisfactory immune response is elicited upto 10 years after the MMR immunization, there is reduction in both the measles seropositivity rates and the measles antibody geometric mean concentrations [3]. An Italian study reported that significant proportion of subjects immunized for measles do not show a protective IgG titers even 10 years after vaccination [4].

Spike (S) of coronavirus and Hemagglutinin $(\mathrm{H})$ of measles virus have a critical involvement in receptor recognition, as well as virus attachment and entry. The $\mathrm{H}$ of measles virus plays a crucial role in success of measles vaccine and the $\mathrm{S}$ protein of coronavirus is the most promising and studied candidate antigen for SARS-CoV vaccine development. It is the major target for neutralizing antibodies in human patients and in animal models. These similarities in structural construct may play a role in eliciting immune response against coronavirus in a child previously immunized against measles.

Age-related declining immunogenicity of measles vaccine, possible structural and functional similarities between measles virus and SARS-CoV-2, sparing of young population from the clinically symptomatic cohort, and importantly, no other plausible immunological explanation of COVID-19 being a predominantly adult age group disease warrants serious probing of measles vaccine as a containment strategy during this ongoing pandemic. Measles vaccination carries a number of advantages: highly efficient, safe, easily manufactured at large scale, vaccine strains are genetically stable, measles does not recombine or integrate genetic material, vaccine does not persist or diffuse, mass booster doses can be given to both pediatric and adult population, and it presents an economical option that can be evaluated swiftly in times of crisis.

Funding: Not Applicable; Competing interest: None stated.

VARNIT SHANKER Consultant Pediatricain and Neonatologist, DACH, Shastri Nagar Road, Jaipur, 302016, India. vas500@g.harvard.edu

\section{REFERENCES}

1. Dowell SF, Ho MS. Seasonality of infectious diseases and severe acute respiratory syndrome - what we don't know can hurt us. Lancet Infect Dis. 2004;4:704-8

2. Thabet F, Chehab M, Bafaqih H, Al Mohaimeed S. Middle East respiratory syndrome coronavirus in children. Saudi Med J. 2015;36:484-6.

3. Carryn S, Feyssaguet M, Povey M, Di Paolo E. Long-term immunogenicity of measles, mumps and rubella-containing vaccines in healthy young children: A 10-year follow-up. Vaccine.2019; 37:5323-31.

4. Bianchi FP, Stefanizzi P, De Nitto S, Larocca A, Germinario C, Tafuri S. Long-term immunogenicity of measles vaccine: An Italian retrospective cohort study. J Infect Dis. 2020;221:721-8. 